\title{
DISPONIBILIDADE DE POTÁSSIO E SUAS RELAÇÕES COM CÁLCIO E MAGNÉSIO EM SOJA CULTIVADA EM CASA-DE-VEGETAÇÃO'
}

\author{
Fábio Alvares de Oliveira ${ }^{2,5 *}$; Quirino Augusto de Camargo Carmello ${ }^{3}$; Hipólito Assunção Antonio \\ Mascarenhas ${ }^{4,5}$ \\ ${ }^{2}$ Depto. de Solos e Engenharia Rural - UFMT/FAMEV - Av. Fernando Corrêa da Costa, s/n, CEP: 78060 -900 \\ Cuiabá, MT. \\ ${ }^{3}$ Depto. de Solos e Nutrição de Plantas - USP/ESALQ, C.P. 9 - CEP: 13418-900 - Piracicaba, SP. \\ ${ }_{5}^{4}$ Centro de Plantas Graníferas - IAC, C.P. 28 - CEP: 13001-970 - Campinas, SP. \\ ${ }^{5}$ Bolsista CNPq. \\ *Autor correspondente <oliveira_fa@hotmail.com>
}

\begin{abstract}
RESUMO: A falta de resposta da soja à aplicação de $\mathrm{K}$ em elevadas quantidades pode estar relacionada com suas interações com o $\mathrm{Ca}$ e o $\mathrm{Mg}$ do solo e, portanto, com a calagem. O objetivo deste trabalho foi avaliar a nutrição potássica da soja em relação ao cálcio e ao magnésio do solo, por um experimento em vasos protegidos, sob casa-de-vegetação, utilizando-se um LATOSSOLO VERMELHO AMARELO distrófico típico, textura média, disposto num esquema fatorial $5 \times 7$ em blocos casualizados, com 4 repetições. Aplicaram-se, 40 dias antes da semeadura, cinco níveis de calcário dolomítico calcinado $\left(0,500,1000,1500,2000 \mathrm{mg} \mathrm{dm}^{-3}\right)$, combinados com sete níveis de $\mathrm{K}\left(0,15,30,45,60,75,90 \mathrm{mg} \mathrm{dm}^{-3}\right)$, aplicados na forma de $\mathrm{KCl}$, previamente à semeadura. Em cada vaso, cultivaram-se 4 plantas de soja inoculadas com Bradyrhizobium japonicum, cultivar precoce IAC-17, com 112 dias de ciclo. Avaliaram-se duas plantas de cada vaso no estádio R2 (florescimento pleno) e duas no estádio R8 (maturação plena). Quando a relação ( $\mathrm{Ca}+\mathrm{Mg}) / \mathrm{K}$ trocável no solo foi superior a 36 ou a relação dos teores foliares $(\mathrm{Ca}+\mathrm{Mg}) / \mathrm{K}$ superior a 3,6, foram verificadas as menores produções de material vegetal em plantas com sintomas de deficiência e teores foliares reduzidos de K. Por outro lado, as maiores produções, aliadas ao maior equilíbrio dos teores foliares de $\mathrm{K}, \mathrm{Ca}$ e $\mathrm{Mg}$ foram obtidas quando a relação $(\mathrm{Ca}+\mathrm{Mg}) / \mathrm{K}$ trocável no solo apresentava-se entre 20 e 30. A relação $(\mathrm{Ca}+\mathrm{Mg}) / \mathrm{K}$ trocável no solo mostrou-se um índice importante de avaliação da disponibilidade do K no solo para a cultura da soja. A recomendação de adubação potássica para a cultura da soja também deve considerar a quantidade de calcário aplicada.
\end{abstract}

Palavras-chave: Glycine max, potássio, material seco, relação $(\mathrm{Ca}+\mathrm{Mg}) / \mathrm{K}$

\section{POTASSIUM AVAILABILITY AND ITS RELATION TO CALCIUM AND MAGNESIUM IN GREENHOUSE CULTIVATED SOYBEAN}

\begin{abstract}
Soybean does not respond well to high levels of $K$, because its availability has been related to interactions with $\mathrm{Ca}$ and $\mathrm{Mg}$ in the soil and, therefore, to liming. In order to study soybean $\mathrm{K}$ nutrition related to soil levels of $\mathrm{Ca}$ and $\mathrm{Mg}$, a greenhouse experiment was carried out in pots with a sandy loam Typic Hapludox. A $5 \times 7$ factorial randomized block experimental design was adopted with four replications. Forty days before sowing, five levels of burned dolomite lime $\left(0,500,1000,1500,2000 \mathrm{mg} \mathrm{dm}^{-3}\right)$ were applied, mixed with seven $\mathrm{K}$ levels $\left(0,15,30,45,60,75,90 \mathrm{mg} \mathrm{dm}^{-3}\right)$ in the form of $\mathrm{KCl}$, before sowing. Four plants were grown in each pot. Plants of the early blooming soybean cultivar, IAC-17 with 112-day cycle, were inoculated with Bradyrhizobium japonicum. Two plants were evaluated at the R2 stage (full bloom) and the other two at the R8 stage (full maturity). When the soil ratio of exchangeable $(\mathrm{Ca}+\mathrm{Mg}) / \mathrm{K}$ was greater than 36 or leaf $(\mathrm{Ca}+\mathrm{Mg}) / \mathrm{K}$ concentration greater than 3.6, the plants presented lower dry matter yield, $\mathrm{K}$ deficiency symptoms and lower leaf $\mathrm{K}$ concentration. Otherwise, greater dry matter yield, related to the optimum equilibrium of leaf $\mathrm{K}, \mathrm{Ca}$ and $\mathrm{Mg}$ concentrations, were verified when the soil ratio of exchangeable $(\mathrm{Ca}+\mathrm{Mg}) / \mathrm{K}$ was between 20 to 30 . The soil ratio of exchangeable $(\mathrm{Ca}+\mathrm{Mg}) / \mathrm{K}$ presented an important index to evaluate soil $\mathrm{K}$ availability for the soybean crop. Recommendations regarding $\mathrm{K}$ fertilization to the soybean crop must also consider the applied liming rates.

Key words: Glycine max, potassium, dry matter, $(\mathrm{Ca}+\mathrm{Mg}) / \mathrm{K}$ ratio
\end{abstract}

\section{INTRODUÇÃO}

Com exceção do nitrogênio, fixado simbioticamente, o potássio é o nutriente mais extraído pela soja e o que se apresenta em maiores concentrações nos tecidos
(Tanaka \& Mascarenhas, 1992). O período de maior exigência do K ocorre no estádio de crescimento vegetativo da soja, apresentando a velocidade de absorção máxima do nutriente nos trinta dias que antecedem ao florescimento (Bataglia \& Mascarenhas, 1977). 
Apesar dessa elevada exigência, são poucos os trabalhos em que se observam respostas dessa cultura à adubação potássica. Os fatores que determinam a ausência deste efeito estão relacionados ao tipo de solo, ao nível do nutriente no solo, a exigência nutricional do cultivar e o tempo de duração reduzido dos experimentos, além da aplicação inadequada do fertilizante (Yamada \& Borkert, 1992).

A falta de resposta à adubação potássica determinou a adoção de práticas pelos produtores, que resultaram na redução dos teores de $\mathrm{K}$ disponível nos solos cultivados com soja. Os teores de $\mathrm{K}$ trocável nos solos que apresentavam-se, em sua maioria, médios a altos e superiores a $1,2 \mathrm{mmol}_{\mathrm{c}} \mathrm{dm}^{-3}$ de K (Gargantini et al. 1970), foram se reduzindo, principalmente, devido a diminuição da quantidade de $\mathrm{K}$ utilizada nos programas de fertilização da cultura e ao aumento da extração de $\mathrm{K}$ do solo, gerado pelo melhoramento genético dos cultivares para aumento de produtividade e ao uso intensivo da terra pelos programas de cultivos sucessivos (Mascarenhas et al., 1987).

A redução dos teores de $\mathrm{K}$ disponível, em áreas sob cultivos sucessivos de soja, tem ocorrido mesmo quando quantidades de 33 a $66 \mathrm{~kg} \mathrm{ha}^{-1}$ de $\mathrm{K}$ têm sido aplicadas anualmente (Borkert et al., 1997a; Mascarenhas et al., 1981; Rosolem et al., 1984;). Ainda assim, dependendo do tipo de solo e dos teores de K no solo, a cultura da soja não tem apresentado respostas à produção de grãos, para as aplicações de K elevadas, apesar de serem verificados aumentos na concentração de $\mathrm{K}$ nas folhas e nos grãos (Borkert et al., 1993b; Borkert et al., 1997a).

A utilização do teor de $\mathrm{K}$ trocável como fator de avaliação do $\mathrm{K}$ disponível do solo pode não ser adequado para a recomendação da adubação potássica. Devido a elevada mobilidade do nutriente no solo, este índice utilizado isoladamente não representa de maneira eficiente a disponibilidade do $\mathrm{K}$ em solos com características mineralógicas, físicas e químicas distintas (Meurer \& Anghinoni, 1993). Além disso, as plantas podem utilizar-se de formas de $\mathrm{K}$ não trocáveis, liberadas durante o ciclo da cultura (Rosolem \& Nakagawa, 1985; Rosolem et al., 1988; Rosolem et al., 1993). Todo esse processo dinâmico do $\mathrm{K}$ nos solos tem como consequência uma diversidade de valores críticos de $\mathrm{K}$ trocável, encontrados na literatura, para a obtenção da produção relativa máxima da soja (Borkert et al., 1993a; Mascarenhas et al., 1981; Raij \& Mascarenhas, 1976).

A disponibilidade de $\mathrm{K}$ no solo e a sua absorção pelas plantas parecem estar relacionadas com a disponibilidade dos cátions divalentes, $\mathrm{Ca}$ e $\mathrm{Mg}$, dominantes do complexo de troca. Ainda assim, a absorção do $\mathrm{K}$ pelas plantas é favorecida em comparação com outras espécies catiônicas sendo, dentre os cátions macronutrientes, o que se apresenta, em geral, em menor e maior concentração no solo e na planta, respectivamente.
A calagem promove 0 aumento das concentrações de $\mathrm{Ca}$ e $\mathrm{Mg}$ do solo, relativamente à do $\mathrm{K}$, podendo reduzir a absorção de $\mathrm{K}$ pelas raízes e provocar sua deficiência (Goedert et al., 1975). No entanto, a calagem favorece a manutenção do teor de $\mathrm{K}$ trocável do solo, pois aumenta a CTC efetiva e reduz as perdas por lixiviação. Em certos casos, pode aumentar a disponibilidade de $\mathrm{K}$ às plantas mais do que a de $\mathrm{Ca}$ e $\mathrm{Mg}$, devido ao menor grau de atração do $\mathrm{K}$ pelas cargas negativas do solo (Barber \& Humbert, 1963). Contudo, a relação entre os teores disponíveis de $(\mathrm{Ca}+\mathrm{Mg})$ e de $\mathrm{K}$ não deve ser muito elevada (Usherwood, 1982).

O balanço ideal de cátions no complexo de troca deve estar ocupado por $65 \%$ de $\mathrm{Ca}^{2+}, 10 \%$ de $\mathrm{Mg}^{2+}, 5 \%$ de $\mathrm{K}^{+}$e $20 \%$ de $\mathrm{H}^{+}$, perfazendo uma saturação igual a $80 \%$ (Bear \& Toth, 1948). No entanto, essa proporção é variável com a cultura, o tipo de solo e também com 0 nível de saturação por bases. A resposta da soja à fertilização potássica tem se mostrado dependente da relação $(\mathrm{Ca}+\mathrm{Mg}) / \mathrm{K}$ trocável nos solos, alcançando as produtividades mais elevadas quando seus valores encontravam-se entre 22 e 31 (Mascarenhas et al., 1987; Mascarenhas et al., 1988; Mascarenhas et al., 2000) ou menores que 40 (Rosolem et al., 1992). Contudo, a correlação entre o $(\mathrm{Ca}+\mathrm{Mg}) / \mathrm{K}$ trocável no solo e a produção de matéria seca pelas plantas não tem apresentado resultados satisfatórios para solos e culturas distintos (Usherwood, 1982), inclusive para a soja (Rosolem et al., 1992).

Os parâmetros que descrevem as relações entre os cátions, para os fatores quantidade e intensidade, são complementares na avaliação adequada do sistema soloplanta (Fassbender, 1972). Devido à relação dos cátions e à elevada mobilidade do $\mathrm{K}$ no solo, a utilização dos teores trocáveis e indicadores do fluxo difusivo de $\mathrm{K}$ podem, conjuntamente, representar melhor a disponibilidade deste nutriente às plantas (Meurer \& Anghinoni, 1993). Portanto, a relação (Ca+Mg)/K trocável nos solos, assim como o potencial potássio-cálciomagnésio $(\mathrm{pK}-1 / 2 \mathrm{p}(\mathrm{Ca}+\mathrm{Mg})$ ) devem se mostrar mais adequados que o teor de $\mathrm{K}$ trocável para os estudos sobre a nutrição potássica.

O trabalho teve por objetivo avaliar a resposta das plantas de soja à aplicação de $\mathrm{K}$ em função do nível de calcário aplicado e identificar, através de correlações, os índices de avaliação de $\mathrm{K}$ no solo mais relacionados à sua verdadeira disponibilidade às plantas.

\section{MATERIAL E MÉTODOS}

O experimento foi conduzido em casa-devegetação pertencente ao Departamento de Solos e Nutrição de Plantas da USP/ESALQ, em vasos plásticos de $10 \mathrm{~kg}$ de capacidade. Foi utilizada a terra fina seca ao ar da camada $0-0,20 \mathrm{~m}$ de um LATOSSOLO 
VERMELHO AMARELO Distrófico típico(EMBRAPA SOLOS, 1999) cuja densidade era de $1,27 \mathrm{~g} \mathrm{~cm}^{-3}$. As características químicas apresentadas anteriormente ao início dos tratamentos eram: $\mathrm{pH}\left(\mathrm{CaCl}_{2} 0,01 \mathrm{~mol} \mathrm{~L}^{-1}\right) 4,2$; $10 \mathrm{mg} \mathrm{dm}^{-3}$ de P(resina); $48 \mathrm{~g} \mathrm{dm}^{-3}$ de M.O.; 0,8; 9; $4 \mathrm{e}$ $56 \mathrm{mmol}_{\mathrm{c}} \mathrm{dm}^{-3}$ de $\mathrm{K}, \mathrm{Ca}, \mathrm{Mg}$ e $\mathrm{H}+\mathrm{Al}$, respectivamente. Foram, também, calculados $S=13,8 \mathrm{mmol}_{\mathrm{c}} \mathrm{dm}^{-3}$; CTC $=69,8 \mathrm{mmol}_{\mathrm{c}} \mathrm{dm}^{-3}$ e V $=20 \%$.

Utilizou-se o delineamento experimental em blocos casualizados, distribuindo-se os tratamentos em esquema fatorial $5 \times 7$, com quatro repetições. O primeiro fator condicionou 0 ambiente em termos de saturação por bases e teores de $\mathrm{Ca}$ e $\mathrm{Mg}$, utilizando-se um calcário dolomítico calcinado $(45 \% \mathrm{CaO}+21 \% \mathrm{MgO}$ e PRNT $=130)$ em cinco níveis $(0,500,1000,1500$ e 2000 $\mathrm{mg} \mathrm{dm}^{-3}$ ). O segundo fator constituiu-se da aplicação de $\mathrm{K}$ no solo, fornecido na forma de $\mathrm{KCl}\left(60 \% \mathrm{~K}_{2} \mathrm{O}\right)$, em sete níveis $\left(0,15,30,45,60,75\right.$ e $\left.90 \mathrm{mg} \mathrm{dm}^{-3}\right)$.

Os tratamentos de calagem foram misturados com todo o volume de terra de cada vaso, e incubados por 30 dias na umidade correspondente a $70 \%$ da capacidade de campo. Após esse período, todos os vasos receberam $200 \mathrm{mg} \mathrm{dm}^{-3}$ de fósforo na forma de superfosfato simples moído e, a seguir, foram aplicadas as doses de $\mathrm{K}$ relativas aos respectivos tratamentos, na forma de $\mathrm{KCl}$ em solução. A semeadura da soja foi realizada no dia 14 de novembro de 1998, sete dias após a aplicação do K. As sementes foram inoculadas com estirpes de Bradyrhizobium japonicum. Cultivaram-se quatro plantas de soja do cultivar precoce IAC-17, com 112 dias de ciclo, mantendo-se a umidade do solo ao redor de $70 \%$ da capacidade de campo, com reposição diária da quantidade de água evapotranspirada, controlada pela pesagem dos vasos.

O acompanhamento do estado nutricional das plantas foi feito com a coleta de duas plantas de cada vaso no florescimento pleno, estádio R2 (Fehr \& Caviness, 1977), divididas em folha diagnóstica (+3), folhas e hastes. Por ocasião da maturação dos grãos, estádio R8 (Fehr \& Caviness, 1977), as outras duas plantas restantes de cada vaso foram coletadas e separadas em folhas, hastes, grãos e vagens. Após 0 preparo das amostras e a avaliação do material seco produzido, foram realizadas as análises químicas dos teores de $\mathrm{K}$, Ca e Mg, apresentadas em g kg${ }^{-1}$, conforme a metodologia descrita por Sarruge \& Haag (1974).

Amostras de terra de cada vaso foram coletadas por ocasião da semeadura. Cada amostra de terra foi composta de cinco subamostras coletadas em pontos distintos do vaso, utilizando-se o amostrador de terra tipo sonda. As amostras foram analisadas segundo a metodologia IAC, proposta por Raij \& Quaggio (1983).

Uma parte de cada amostra de terra coletada foi utilizada para a extração da solução do solo na umidade de $70 \%$ da capacidade de campo. A extração da solução do solo foi realizada pelo método da centrifugação modificado (Elkhatib et al., 1987) e adaptado. Foi utilizado, para a extração, um conjunto de dois tubos superpostos, sendo a terra acondicionada no primeiro, ajustado internamente ao segundo. A solução do solo foi extraída por um orifício central, protegido por tela, na base inferior desse tubo e coletada no segundo tubo, durante 30 minutos de centrifugação a uma velocidade de rotação de 8800 rpm (13000 g). Foram determinados, na solução do solo extraída, o pH, a condutividade elétrica (CE) e, as concentrações de $\mathrm{K}$ (fotometria de chama), de $\mathrm{Ca}$ e de $\mathrm{Mg}$ (espectrofotometria de absorção atômica).

A estimativa da força iônica $(\mu)$ da solução do solo, corrigida para pares de íons, foi calculada utilizando-se a relação $\mu \times$ CE (Gillman \& Bell, 1978). Com os valores obtidos para as concentrações do $\mathrm{K}$, do $\mathrm{Ca}$ e do Mg, presentes na solução do solo, e da estimativa da força iônica, foram calculados os coeficientes de atividade (f) do $\mathrm{K}$, do $\mathrm{Ca}$ e do $\mathrm{Mg}$, de acordo com a fórmula completa de Debye-Hückel, descrita por Wolt (1994):

$$
\log f=\frac{-0,509 \cdot Z^{2} \cdot \mu^{1 / 2}}{1+0,33 \cdot a_{i}^{0} \cdot \mu^{1 / 2}} ;
$$

em que $Z$ é a valência do íon, $a_{i}{ }^{0}$ é parâmetro correspondente ao tamanho do íon $\left(10^{-8} \mathrm{~m}^{1} \mathrm{a}_{\mathrm{i}}^{0}=10^{-8}\right.$ metros) e, $\mu$ é a força iônica da solução calculada por:

$$
\mu=1 / 2 \sum_{i=1}^{i=n} m_{i} z_{i}^{2}
$$

sendo $m_{i}$, a concentração molar do íon. $A$ atividade foi dada pela fórmula: $a=f . m_{i}$; resultando nas atividades do $\mathrm{K}(\mathrm{aK})$, do $\mathrm{Ca}(\mathrm{aCa})$ e do $\mathrm{Mg}(\mathrm{aMg})$.

O potencial potássio-cálcio-magnésio foi determinado como sendo uma função logarítmica do inverso da relação das suas atividades iônicas (aK/ $\left.(\mathrm{aCa}+\mathrm{aMg})^{1 / 2}\right)$, conforme Fassbender (1972): $\mathrm{p}(\mathrm{Ca}+\mathrm{Mg})$

Potencial de potássio-cálcio-magnésio $=\mathrm{pK}-1 / 2$

A análise estatística dos resultados foi executada através do pacote estatístico SAS (SAS Institute, 1996), aplicando-se o teste $\mathrm{F}$ a $5 \%$ e $1 \%$ para a análise da variância, ajustando-se as variáveis através de regressões linear e quadrática nos casos significativos. Foram determinadas as correlações entre os resultados de análise de terra e os resultados de análise vegetal, avaliadas pelo teste t e selecionadas pelo coeficiente de correlação.

\section{RESULTADOS E DISCUSSÃO}

As plantas de soja apresentaram resposta positiva à aplicação de $\mathrm{K}$, para a produção de material seco da parte aérea, tanto no florescimento (R2) quanto no final do ciclo de maturação (R8), sem, contudo, apresentar interação significativa com a calagem (Figura 1). As produções das folhas e das hastes, até o início do florescimento, foram aumentadas de maneira 
quadrática em $40 \%$, em resposta à aplicação de potássio até a maior dose estudada. Esses valores são muito significativos quando comparados aos aumentos de $15 \%$ verificados em plantas de soja que receberam $60 \mathrm{mg} \mathrm{dm}^{-3}$ de K (Rossetto et al., 1995). Ao final do ciclo, a produção de material vegetativo manteve o aumento de $40 \%$ em resposta ao $\mathrm{K}$, e o material reprodutivo, vagens e grãos, apresentou um aumento relativo de $150 \%$, resultando numa resposta quadrática ao $\mathrm{K}$ aplicado que atingiu um crescimento de $70 \%$ do material seco da parte aérea na maior dose. Em geral, a produção de material seco da parte aérea foi crescente até $30 \mathrm{mg}$ $\mathrm{dm}^{-3}$ de $\mathrm{K}$, seguida de uma tendência de estabilização até o ponto de máxima produção, entre $66 \mathrm{e} 83 \mathrm{mg} \mathrm{dm}^{-3}$.

O aumento elevado na produção de grãos está ligado as maiores diferenças entre os tratamentos para os teores de $\mathrm{K}$ disponível no solo, na fase inicial do desenvolvimento reprodutivo. De acordo com Bataglia \& Mascarenhas (1977), 50 a $60 \%$ do material vegetal e do $\mathrm{K}$ já deveriam ter sido acumulados até essa época, indicando uma redução na disponibilidade de $\mathrm{K}$ no solo que atingiu, no final do ciclo, o valor mínimo de $0,4 \mathrm{mmol}_{\mathrm{c}}$ $\mathrm{dm}^{-3}$ de $\mathrm{K}$ trocável em todos os tratamentos. O aumento da produção de grãos de soja com a adubação potássica tem sido verificado esporadicamente (Rosolem et al., 1984), mas difere da maioria dos resultados da literatura. Nestes, não foram observados aumentos de produção, mesmo naqueles solos que tiveram o teor de $\mathrm{K}$ trocável reduzido ao longo de cultivos sucessivos (Borkert et al., 1997a; Borkert et al., 1997b; Mascarenhas et al., 1981).

A tendência de estabilização da produção de material seco da soja, mesmo com o aumento da disponibilidade de K, é confirmada pelo crescimento linear da quantidade de $\mathrm{K}$ acumulada pela parte aérea das plantas ao final do ciclo de maturação (Figura 2). $O$ aumento do $\mathrm{K}$ disponível no solo intensificou o efeito competitivo sobre a absorção do $\mathrm{Ca}$ e do $\mathrm{Mg}$, sendo mais efetivo sobre esse último, tanto que a quantidade máxima de $\mathrm{Mg}$ acumulada foi verificada quando $41 \mathrm{mg} \mathrm{dm}^{-3}$ de $\mathrm{K}$ foram aplicados (Figura 2), apesar do aumento da produção de material seco até a dose de $78 \mathrm{mg} \mathrm{dm}^{-3}$ de $\mathrm{K}$ (Figura 1). A absorção de Mg pela soja é, portanto, mais uma função do $\mathrm{K}$ disponível do que propriamente do Mg trocável no solo (Rosolem et al., 1992).

Tanto a produção de material seco quanto o acúmulo de $\mathrm{K}$ pela parte aérea foram vinculados ao aumento da disponibilidade do $\mathrm{K}$ no solo. Com a aplicação do nutriente, foram verificadas respostas quadráticas diretamente proporcionais não apenas para o K trocável e a aK, mas também, para a disponibilidade do $\mathrm{K}$ relativa ao $\mathrm{Ca}$ e ao $\mathrm{Mg}$ na fase trocável e na solução do solo (Figuras 3). Contudo, o teor de $\mathrm{K}$ trocável e os índices de avaliação da disponibilidade do $\mathrm{K}$ em solução apresentaram tendência de estabilização a partir da aplicação de quantidades superiores a 60 e $75 \mathrm{mg}$ $\mathrm{dm}^{-3}$ de $\mathrm{K}$, determinando a taxa de acumulação de $\mathrm{K}$ e o aumento de sua concentração nas maiores doses aplicadas. A relação $(\mathrm{Ca}+\mathrm{Mg}) / \mathrm{K}$ trocável do solo, por sua vez, apresentou tendência de estabilização a partir de $45 \mathrm{mg} \mathrm{dm}^{-3}$ de $\mathrm{K}$ indicando maior correlação com a produção de material vegetal.

A maior disponibilidade de $\mathrm{K}$ no solo resultou em aumento do teor de $\mathrm{K}$ nas folhas de 5 para $10,1 \mathrm{~g} \mathrm{~kg}^{-1}$, considerado ainda deficiente para a soja (Ohlrogge \& Kamprath, 1968). Os teores de $\mathrm{K}$ nas folhas relacionados com as maiores produtividades de grãos em solos de cerrado têm sido verificados entre $13 \mathrm{e} 17 \mathrm{~g} \mathrm{~kg}^{-1}$ (Borkert et al., 1993b; Mascarenhas et al., 2000; Ritchey et al., 1987; Rosolem et al., 1984). Devido aos teores reduzidos nas folhas, as plantas manifestaram a deficiência de $\mathrm{K} \mathrm{a}$ partir do estádio V8 (Fehr \& Caviness, 1977), anterior ao início do florescimento. Os sintomas eram característicos nas folhas mais velhas das plantas que receberam doses de $\mathrm{K}$ crescentes até $30 \mathrm{mg} \mathrm{dm}^{-3}$, conforme o aumento do nível de calcário aplicado. A calagem influenciou na disponibilidade do $\mathrm{K}$ no solo, principalmente quando este

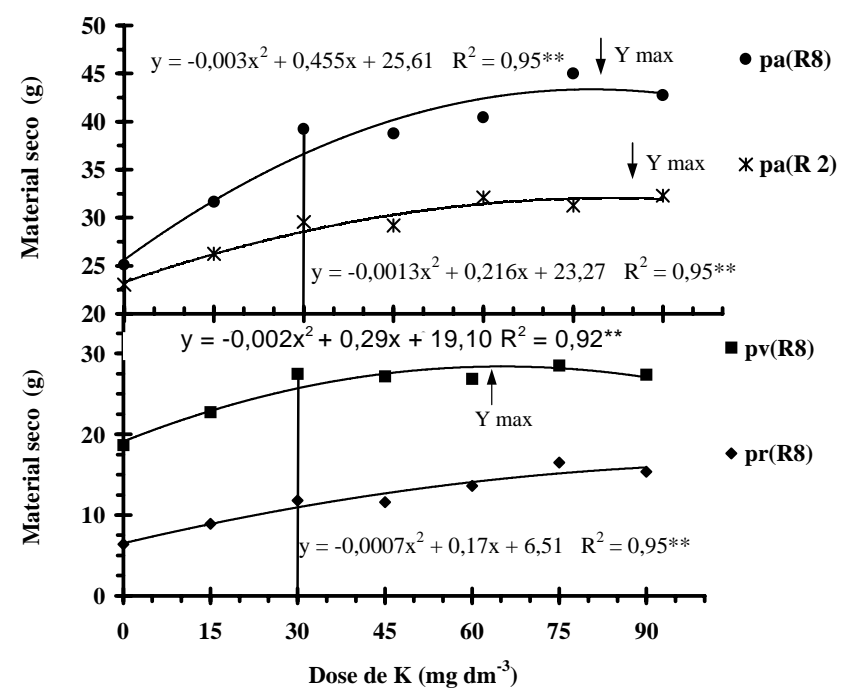

Figura 1 - Produção de material seco da parte vegetativa (pv) e da parte reprodutiva ( $\mathrm{pr}$ ) das plantas de soja avaliadas no estádio $R 8$ e da parte aérea (pa) avaliada nos estádios R2 e R8, em função da aplicação de K e na presença de todos os níveis de calagem.

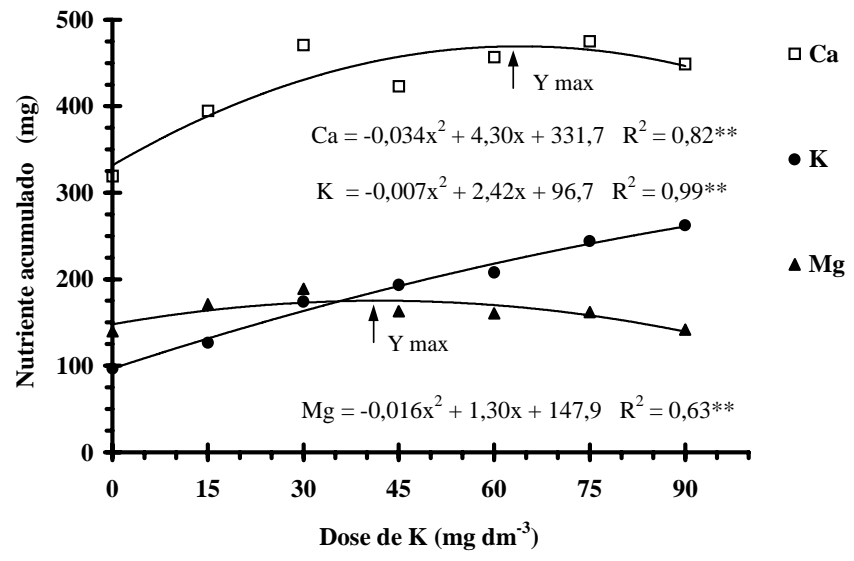

Figura 2 - Acumulação de $\mathrm{K}, \mathrm{Ca}$ e Mg pela parte aérea da soja no estádio R8, em função da aplicação de K e na presença de todos os níveis de calagem. 
nutriente foi aplicado em quantidades de até $15 \mathrm{mg} \mathrm{dm}^{-3}$, resultando em valores médios da relação $(\mathrm{Ca}+\mathrm{Mg}) / \mathrm{K}$ no solo superiores a 36 (Figura 3 ).

Além do teor de $\mathrm{K}$ foliar reduzido, as plantas deficientes apresentaram a relação $(\mathrm{Ca}+\mathrm{Mg}) / \mathrm{K}$ nas folhas superior a 3,6 (Figura 4). Valores acima de 3,2 indicam um desbalanço nos teores de $\mathrm{K}$ em relação ao $\mathrm{Ca}$ e ao Mg absorvidos (Mascarenhas et al.,1987). Em condições ótimas de disponibilidade de $\mathrm{K}$, a relação $(\mathrm{Ca}+\mathrm{Mg}) / \mathrm{K}$ nas folhas varia entre 1,07 e 1,4 (Mascarenhas et al., 2000).

As relações $\mathrm{Ca} / \mathrm{K}, \mathrm{Mg} / \mathrm{K}$ e $(\mathrm{Ca}+\mathrm{Mg}) / \mathrm{K}$ nas folhas foram reduzidas com o aumento da disponibilidade do $\mathrm{K}$ nos solos (Figura 4). Confirmando o maior efeito antagônico do $\mathrm{K}$ sobre a absorção de $\mathrm{Mg}$ do que sobre a de $\mathrm{Ca}$, a relação $\mathrm{Ca} / \mathrm{Mg}$ nas folhas aumentou de 2,7 para 3,4 com a aplicação da maior dose. A relação $(\mathrm{Ca}+\mathrm{Mg}) / \mathrm{K}$ nas folhas foi reduzida severamente de 4,0 para 2,4 quando foram aplicados $45 \mathrm{mg} \mathrm{dm}^{-3}$ de K. A partir desse valor a aplicação de $\mathrm{K}$ exerceu uma menor influência sobre essa relação foliar que atingiu o limite mínimo de 1,7.

Da mesma forma que indicam os resultados da literatura (Rosolem et al., 1992; Usherwood, 1982), não foram obtidas correlações elevadas entre os índices de avaliação da disponibilidade de $\mathrm{K}$ no solo e a produção de material seco ou o K na planta (TABELA 1). Ainda assim, a relação $(\mathrm{Ca}+\mathrm{Mg}) / \mathrm{K}$ trocável apresentou uma maior correlação com a produção de material seco no estádio $\mathrm{R} 8$ do que o $\mathrm{K}$ trocável e os índices de disponibilidade de $\mathrm{K}$ em solução, que se mostraram mais relacionados com a quantidade de $\mathrm{K}$ acumulada pela parte aérea. As correlações da produção com os teores e as relações entre o $\mathrm{K}$, ० $\mathrm{Ca}$ e o $\mathrm{Mg}$ nas folhas também não se mostraram significativas. A menor correlação do $\mathrm{K}$ trocável e dos índices de $\mathrm{K}$ em solução com a produção de matéria seca pela parte aérea indicam que a resposta da soja à adubação potássica apresenta-se dependente de outros fatores, como os teores trocáveis de $\mathrm{Ca}$ e de $\mathrm{Mg}$.

As maiores produções de matéria seca da parte aérea, verificadas quando o $\mathrm{K}$ foi aplicado em quantidades superiores a $30 \mathrm{mg} \mathrm{dm}^{-3}$ (Figura 1), ocorreram quando os teores de $\mathrm{K}$ trocável no solo foram superiores a $1,6 \mathrm{mmol}_{\mathrm{c}} \mathrm{dm}^{-3}$ e a relação $(\mathrm{Ca}+\mathrm{Mg}) / \mathrm{K}$ trocável variou entre 20 e 30, independente do nível de calcário aplicado (Figura 3). Nessas condições, quando a influência da aplicação de $K$ sobre a produção foi reduzida, o aumento da disponibilidade do $\mathrm{K}$ no solo resultou em aumento na concentração e no acúmulo de $\mathrm{K}$ nos tecidos foliares (Figuras 2 e 4). Assim, dentro da faixa ideal da relação $(\mathrm{Ca}+\mathrm{Mg}) / \mathrm{K}$ no solo, a aplicação de $\mathrm{K}$ alterou o balanço nutricional, porém, não refletiu em prejuízos ou ganhos para o crescimento vegetativo.

Quando os valores da relação $(\mathrm{Ca}+\mathrm{Mg}) / \mathrm{K}$ trocável no solo estavam acima de 30 , o desbalanço nutricional foi evidenciado pelas acentuadas reduções das relações foliares do $\mathrm{Ca}$ e do $\mathrm{Mg}$ com o K em função da aplicação de $\mathrm{K}$. Dentro dos limites da relação $(\mathrm{Ca}+\mathrm{Mg}) / \mathrm{K}$ trocável no solo que resultaram nas maiores produções, verificou-se que a relação $(\mathrm{Ca}+\mathrm{Mg}) / \mathrm{K}$ nas folhas esteve sempre abaixo de 3,0 e apresentou-se menos influenciada pela adubação potássica (Figura 4). Essa redução nas variações das relações dos teores foliares pode indicar a faixa adequada de $\mathrm{K}$ às plantas (Mascarenhas et al., 2000) mas, também indica que o equilíbrio nutricional entre o $\mathrm{K}$, ० $\mathrm{Ca}$ e $\circ \mathrm{Mg}$ está diretamente relacionado com a relação $(\mathrm{Ca}+\mathrm{Mg}) / \mathrm{K}$ trocável no solo.
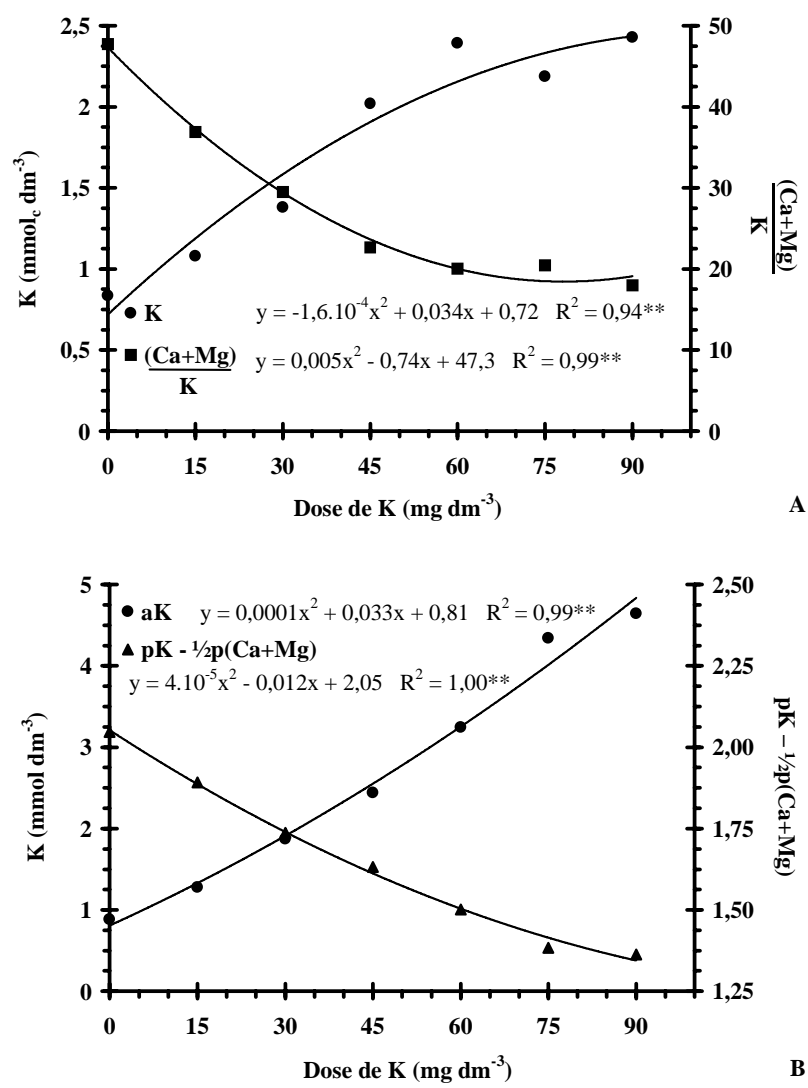

Figura 3 - Teores trocáveis de $\mathrm{K}$ e relação $(\mathrm{Ca}+\mathrm{Mg}) / \mathrm{K}(\mathrm{a})$, aK e potencial K-Ca-Mg na solução do solo (b), no período anterior à semeadura da soja, em função da aplicação de potássio e na presença de todos os níveis de calagem.

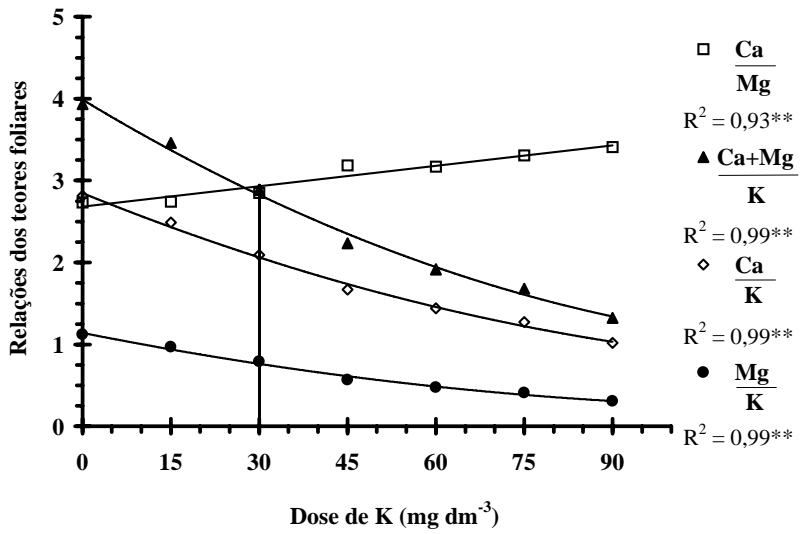

Figura 4 - Relações Ca/Mg, $(\mathrm{Ca}+\mathrm{Mg}) / \mathrm{K}, \mathrm{Ca} / \mathrm{K}$ e $\mathrm{Mg} / \mathrm{K}$ nas folhas +3 de soja, em função da aplicação de potássio e na presença de todos os níveis de calagem. 
TABELA 1 - Coeficientes de correlação para os modelos matemáticos que relacionam os índices de nutrição potássica e de produção da soja, avaliados nos estádios $\mathrm{R} 2$ e R8 ${ }^{1}$, com os índices de disponibilidade do $\mathrm{K}$ no solo, no período anterior à semeadura.

\begin{tabular}{|c|c|c|c|c|c|}
\hline \multirow[t]{2}{*}{ Índice de $\mathrm{K}$ do solo } & \multicolumn{2}{|c|}{ Material seco da parte aérea } & \multirow{2}{*}{$\begin{array}{c}\text { Folha diagnóstica } \\
\text { Estádio R2 }\end{array}$} & \multicolumn{2}{|c|}{$\mathrm{K}$ acumulado pela parte aérea } \\
\hline & Estádio R2 & Estádio R8 & & Estádio R2 & Estádio R8 \\
\hline \multicolumn{6}{|l|}{ Fase trocável } \\
\hline \multirow[t]{2}{*}{$\mathrm{K}$} & $Y=a+b / x$ & $Y=a+b x+c x^{2}$ & $Y=a+b x+c x^{2}$ & $Y=a+b / x$ & $Y=a+b x+c x^{2}$ \\
\hline & $\mathrm{R}=0,23^{* *}$ & $R=-0,21 n s$ & $\mathrm{R}=0,53^{* *}$ & $r=0,64^{* *}$ & $\mathrm{R}=0,37^{* *}$ \\
\hline \multirow[t]{2}{*}{$\frac{\mathrm{Ca}+\mathrm{Mg}}{\mathrm{K}}$} & $Y=a+b / x$ & $Y=a+b x+c x^{2}$ & $Y=a+b x+c x^{2}$ & $Y=a+b x$ & $Y=a+b x+c x^{2}$ \\
\hline & $\mathrm{R}=0,28^{* *}$ & $\mathrm{R}=0,47^{* *}$ & $\mathrm{R}=-0,71^{\star *}$ & $r=-0,47^{* *}$ & $R=-0,26^{* *}$ \\
\hline \multicolumn{6}{|l|}{ Solução do solo } \\
\hline \multirow[t]{2}{*}{ a K } & $Y=a+b x+c x^{2}$ & $Y=a+b x+c x^{2}$ & $Y=a+b x+c x^{2}$ & $Y=a+b x+c x^{2}$ & $Y=a+b x+c x^{2}$ \\
\hline & $\mathrm{R}=0,37^{* *}$ & $R=-0,32^{* *}$ & $\mathrm{R}=0,61^{* *}$ & $\mathrm{R}=0,83^{* *}$ & $\mathrm{R}=0,61^{* *}$ \\
\hline \multirow[t]{2}{*}{ pK - 1/2p(Ca+Mg) } & $Y=a+b x+c x^{2}$ & $Y=a+b x+c x^{2}$ & $Y=a+b x+c x^{2}$ & $Y=a+b x+c x^{2}$ & $Y=a+b x+c x^{2}$ \\
\hline & $R=-0,32^{* *}$ & $R=-0,22^{*}$ & $R=-0,69$ ** & $R=-0,78^{* *}$ & $R=-0,51^{* *}$ \\
\hline
\end{tabular}

${ }^{1}$ Fehr \& Caviness (1977). ${ }^{*} e^{* *}$, significativos a $5 \%$ e $1 \%$, respectivamente, pelo teste t. ns, não significativo pelo teste t.

\section{CONCLUSÕES}

Os índices de disponibilidade de $\mathrm{K}$ no solo relativos aos teores de $\mathrm{Ca}$ e de $\mathrm{Mg}$, tanto do complexo de troca quanto da solução do solo, estão diretamente correlacionados com a nutrição potássica da soja.

A relação $(\mathrm{Ca}+\mathrm{Mg}) / \mathrm{K}$ trocável no solo constitui um índice importante de avaliação da disponibilidade do $\mathrm{K}$ no solo para a cultura da soja.

A recomendação de adubação potássica para a cultura da soja deve considerar a quantidade de calcário aplicada.

\section{AGRADECIMENTOS}

À FAPESP, pelo financiamento concedido para a execução do projeto, sob o processo n ${ }^{\circ}$ 98/09816-1.

\section{REFERÊNCIAS BIBLIOGRÁFICAS}

BARBER, S.A.; HUMBERT, R.P. Advances in knowledge of potassium relationship in the soil and plant. In: McVICKAR, M.H.; BRIDGER, G.L.; NELSON, L.B. Fertilizer tecnology and uses. Madison: SSSA, 1963. cap.11, p.231-268.

BATAGLIA, O.C.; MASCARENHAS, H.A.A. Absorção de nutrientes pela soja. Campinas: Instituto Agronômico, 1977. 36p. (Boletim Técnico, 41).

BEAR, F.E.; TOTH, S.J. Influence of calcium on availability of other soil cations. Soil Science, v.65, p.69-74, 1948.

BORKERT, C.M.; FARIAS, J.R.B.; SFREDO, G.J.; TUTIDA, F.; SPOLADORI, C.L. Resposta da soja à adubação e disponibilidade de potássio em LATOSSOLO ROXO Álico. Pesquisa Agropecuária Brasileira, v.32, p.1119-1129, 1997a.

BORKERT, C.M.; FARIAS, J.R.B.; SFREDO, G.J.; TUTIDA, F.; SPOLADORI, C.L. Resposta da soja à adubação e disponibilidade de potássio em LATOSSOLO ROXO Eutrófico. Pesquisa Agropecuária Brasileira, v.32, p.1009-1022, 1997b.
BORKERT, C.M.; SFREDO, G.J.; SILVA, D.N.da. Calibração de potássio trocável para soja em LATOSSOLO ROXO Distrófico. Revista Brasileira de Ciência do Solo, v.17, p.223-226, 1993a.

BORKERT, C.M.; SILVA, D.N.da; SFREDO, G.J. Calibração de potássio nas folhas de soja em LATOSSOLO ROXO Distrófico. Revista Brasileira de Ciência do Solo, v.17, p.227-230, 1993b.

ELKHATIB, E.A.; HERN, J.L.; STALEY, T.E. A rapid centrifugation method for obtaining soil solution. Soil Science Society of America Journal, v.51, p.578-583, 1987.

EMBRAPA SOLOS. Sistema brasileiro de classificação de solos. Brasília: EMBRAPA Produção de Informação, 1999. 412p.

FASSBENDER, H.W. Equilibrio cationicos y disponibilidad de potásio en suelos de América Central. Turrialba, v.22, p.388397, 1972.

FEHR, W.A.; CAVINESS, C.E. Stages of soybean development. Ames: lowa State University, 1977. 11p. (lowa Agriculture Experimental Station Bulletin, 80)

GARGANTINI, H.; COELHO, F.A.S; SOARES, F.V.E. Levantamento de fertilidade dos solos do Estado de São Paulo. Campinas: Instituto Agronômico, 1970. 32p.

GILLMAN, G.P.; BELL, L.C. Soil solution studies on weathered soils from tropical North Queensland. Australian Journal of Soil Research, v.16, p.67-77, 1978.

GOEDERT, W. J.; COREY, R.B.; SYERS, J.K. The effects on potassium equilibria in soils of Rio Grande do Sul, Brazil. Soil Science, v.120, p.107-111, 1975.

MASCARENHAS, H.A.A.; BULISANI, E.A.; MIRANDA, M.A.C; BRAGA, N.R.; PEREIRA, J.C.V.N.A. Deficiência de potássio em soja no Estado de São Paulo: melhor entendimento do problema e possíveis soluções. O Agronômico, v.40, p.3443, 1988.

MASCARENHAS, H.A.A.; MIRANDA, M.A.C; LELIS, L.G.L.; BULISANI, E.A.; BRAGA, N.R.; PEREIRA, J.C.V.N.A. Haste verde e retenção foliar em soja causada por deficiência de potássio. Campinas: Instituto Agronômico, 1987. 15p. (Boletim Técnico, 199).

MASCARENHAS, H.A.A.; TANAKA, R.T.; CARMELLO, Q.A.C.; GALLO, P.B.; AMBROSANO, G.M.B. Calcário e potássio para a cultura da soja. Scientia Agricola, v.57, p.445-449, 2000. 
MASCARENHAS, H.A.A.; VALADARES, J.M.A.; ROTTA, C.L.; BULISANI, E.A. Adubação potássica na produção de soja, nos teores de potássio em LATOSSOLO ROXO Distrófico de cerrado. Bragantia, v.40, p.125-134, 1981.

MEURER, E.J.; ANGHINONI, I. Disponibilidade de potássio e sua relação com parâmetros de solo. Revista Brasileira de Ciência do Solo, v.17, p.377-382, 1993.

OHLROGGE, A.J.; KAMPRATH, E.J. Fertilizer use in soybeans. In: DINAUER, R.C. (Ed.). Changing patterns in fertilizer use. Madison: SSSA, 1968. p.273-295.

RAIJ, B. van; MASCARENHAS, H.A.A. Calibração de potássio e fósforo em solos para soja. In: CONGRESSO BRASILEIRO DE CIÊNCIA DO SOLO, 15., Campinas, 1975. Anais. Campinas: SBCS, 1976. p.309-315.

RAIJ, B. van; QUAGGIO, J.A. Métodos de análise de solo para fins de fertilidade. Campinas: Instituto Agronômico, 1983. 31p. (Boletim Técnico, 81).

RITCHEY, K.D.; CERKAUSKAS, R.F.; SILVA, J.U.; VILELA, L. Residual effects of potassium and magnesium on soybean yield and on disease incidence in a cerrado dark red latosol. Pesquisa Agropecuária Brasileira, v.22, p.825-832, 1987.

ROSOLEM, C.A.; BESSA, A.M.; PEREIRA, H.F.M. Dinâmica do potássio no solo e nutrição potássica da soja. Pesquisa Agropecuária Brasileira, v.28, p.1045-1054, 1993.

ROSOLEM, C.A.; MACHADO, J.R.; MAIA, L.C.; NAKAGAWA, J. Respostas de soja ao magnésio do solo. Revista Brasileira de Ciência do Solo, v.16, p.47-54, 1992.

ROSOLEM, C.A.; MACHADO, J.R.; RIBEIRO, D.B.O. Formas de potássio no solo e nutrição potássica da soja. Revista Brasileira de Ciência do Solo, v.12, p.121-125, 1988.

ROSOLEM, C.A.; NAKAGAWA, J. Potassium uptake by soybean as affected by exchangeable potassium in soil. Communication in Soil Science and Plant Analysis, v.16, p.707-726, 1985.
ROSOLEM, C.A.; NAKAGAWA, J.; MACHADO, J.R. Adubação potássica da soja em LATOSSOLO VERMELHO ESCURO fase arenosa. Pesquisa Agropecuária Brasileira, v.19, p.1319-1326, 1984

ROSSETTO, C.A.V.; FERNANDES, D.M.; ISHIMURA, I.; ROSOLEM, C.A. Diferentes respostas de cultivares de soja ao potássio. Pesquisa Agropecuária Brasileira, v.30, p.1225-1231, 1995.

SARRUGE, J.R.; HAAG, H.P. Análises químicas em plantas. Piracicaba: USP/ESALQ, Depto. de Química, 1974. 56p.

SAS INSTITUTE. The SAS-system for windows: release 6.11 (software). Cary: Statistical Analysis System Institute, 1996.

TANAKA, R.T.; MASCARENHAS, H.A.A. Soja, nutrição, correção do solo e adubação. Campinas: Fundação Cargill, 1992. 60p. (Série Técnica, 7)

USHERWOOD, N.R. Interação do potássio com outros íons. In: SIMPÓSIO SOBRE O POTÁSSIO NA AGRICULTURA BRASILEIRA, Londrina, 1982. O potássio na agricultura brasileira. Piracicaba: Potafos, 1982. p.227-247.

WOLT, J.D. Chemical statics and dynamics applied to soil solution. In: WOLT, J.D. Soil solution chemistry: applications to environmental science and agriculture. New York: John Wiley \& Sons, 1994. cap.3, p.22-73.

YAMADA, T; BORKERT, C.M. Nutrição e produtividade da soja. In: SIMPÓSIO SOBRE CULTURA E PRODUTIVIDADE DA SOJA, 1., Piracicaba, 1992. Anais. Piracicaba: USP/ESALQ, 1992. p.180-212.

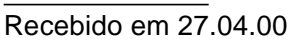

